\title{
El papel del espía y la maleabilidad de la trama en Our Man in Havana de Graham Greene
}

\section{The role of the spy and the adaptability of the plot in Our Man in Havana by Graham Greene}

\author{
BLANCA HERRMANN ESTUdILlO \\ Universidad Nacional Autónoma de México \\ blanca.herrmann28@gmail.com \\ ORCID ID: 0000-0002-4993-5670
}

\begin{abstract}
Resumen: Our Man in Havana (1958), de Graham Greene, y sus adaptaciones (cinematográfica en 1959 , por Carol Reed; y teatral en 2009, por Clive Francis) emplean la sátira para hacer una burla a la trama de espías. Se hará un estudio de estas obras centrado en la representación de la figura del espía en la novela, y cómo el protagonista es un móvil que enfatiza el aspecto histórico del espacio en la película. El análisis de la obra teatral, con respecto al protagonista y el espacio, expondrá qué cambios ocurren al suprimir aspectos importantes del contexto histórico. Así, las dos primeras obras exhiben instancias diferentes que emplean la sátira para resaltar los defectos de la sociedad inglesa en cuanto al espionaje. La tercera diluye el contexto y presenta una trama que se acerca más a la farsa.
\end{abstract}

Palabras clave: adaptación, sátira, farsa, Guerra Fría, ficción de espías, Our Man in Havana.

\begin{abstract}
Our Man in Havana (1958), by Graham Greene, and its adaptations (the 1959 film, by Carol Reed; and the 2009 theatrical version, by Clive Francis) use satire as a means to mock the spy plot in fiction. The analysis of these works will focus on the figure of the spy in the novel and how the protagonist is a mobile that emphasizes the historical aspect of the space in the film. The analysis of the play, with respect to the protagonist and the space, will show what changes occur at the level of the plot by suppressing important aspects of the historical context. Thus, the first two works express different instances that employ satire to make a comment on the faults of English society in terms of espionage. The third one dilutes the context to present a plot closer to the farce.
\end{abstract}

Keywords: adaptation, satire, farce, cold war, spy fiction, Our Man in Havana. 


\section{INTRODUCCIÓN: LA GUERRA FRÍA, LA ADAPTACIÓN Y LA OBRA DE GRAHAM GREENE}

Ambientada en los años cincuenta, Our Man in Havana (1958), de Graham Greene, usa la sátira como medio principal para hacer un comentario burlesco de la tensión social que generaba la Guerra Fría. Las intrigas y tensión entre naciones durante este periodo fueron las razones por las que Greene plantea una narración en la que un hombre obligado a trabajar para el servicio secreto británico crea una red de mentiras mediante una serie de reportes falsos que hacen que las grandes potencias dirijan su atención a Cuba. Así, debido a las fantasías del protagonista, la brecha que divide la verdad y la ficción dentro de la diégesis quedará difuminada. El propósito de este ensayo, por tanto, es hacer un estudio sobre la adaptación como proceso de cambio y reinterpretación de un texto literario. Linda Hutcheon (2013: 7) argumenta sobre este tema en $A$ Theory of Adaptation:

An adaptation is an announced and extensive transposition of a particular work or works. This 'transcoding' can involve a shift of medium (a poem to a film) or genre (an epic to a novel), or a change of frame and therefore context: telling the same story from a different point of view, for instance, can create a manifestly different interpretation.

Así, la adaptación será producto de la transposición de una obra de un medio a otro. Este movimiento implica la re-contextualización o variación de distintos aspectos; por ejemplo, cambio de escenario o contexto histórico, personajes, punto de vista narrativo, etc. Es decir, la adaptación es un proceso de creación en el que se lleva a cabo la reinterpretación de una obra base. De esta manera, Our Man in Havana servirá como el objeto principal de estudio para poner en práctica el análisis de la adaptación como un medio que propicia la sobrevivencia de un texto en distintas expresiones artísticas, aparte de la literaria. La adaptación cinematográfica y teatral de la novela serán estudiadas con base en el protagonista de la historia, Jim Wormold, para demostrar cómo evoluciona este personaje en cada una de sus representaciones.

Our Man in Havana gira en torno a Jim Wormold, un hombre inglés radicado en Cuba que vive con su joven hija, Milly. El protagonista es un vendedor de aspiradoras que constantemente tiene problemas económicos debido a la lujosa vida que lleva la adolescente. Por azares del destino es reclutado como agente del servicio secreto británico y obligado por su superior a reportar toda actividad sospechosa que pueda desatar una guerra entre el bloque socialista y 
capitalista. Wormold acepta el trabajo ya que le ofrecen una considerable cantidad de dinero. Sin embargo, para justificar los gastos, el protagonista crea un entramado de reportes ficticios -entre los que destacan la construcción de misiles en Cuba e incluso la contratación de subagentes imaginarios que lo ayudan a conseguir información-. Las mentiras comienzan a levantar sospechas en Inglaterra, por lo que, la atención del servicio secreto cae sobre él. La muerte de hombres inocentes, debido a las mentiras de Wormold, lo obligan a abandonar Cuba. De regreso en Inglaterra, el servicio secreto lo absuelve para evitar la vergüenza que causaría la revelación de que las mentiras de un hombre pudieron haber causado una nueva guerra.

El contexto histórico en el que se desenvuelve Our Man in Havana es la base que sustenta el eje satírico de la obra. Mientras que la novela nunca menciona una fecha exacta en la que se desarrolla la trama, una de las escenas iniciales del filme muestra la leyenda: «This film is set in Cuba before the recent revolution» (Ver fot. 1). Debido a esto, la trama engloba un contexto político y social específicos de la Guerra Fría y el inicio de la Revolución Cubana, es decir, un periodo de finales de la década de los años cincuenta. Las grandes potencias mundiales -Inglaterra, Estados Unidos y la Unión Soviéticaluchaban por el liderazgo político e ideológico después de la Segunda Guerra Mundial. Al igual que Estados Unidos, durante los años posteriores a este conflicto armado, Inglaterra había tomado la decisión de desarrollar armas nucleares en caso de algún ataque del bando comunista. Sin embargo, desde finales de la guerra, el Imperio británico sufrió un proceso de descolonización y su poder económico se vio reducido en comparación con la ascendente economía de los Estados Unidos.

La creación de armas nucleares fue una de las formas de demostrar poder ante las potencias globales, por lo que la información y las noticias sobre lo que hacían los bandos enemigos eran de gran importancia para monitorear el poder de alcance de los países protagonistas de la Guerra Fría. Sobre este tema, argumenta Anne Deighton: «Cold War exigencies required efforts to monitor Communist activity and weed Communists out of public services and trades unions at home and abroad, given the perception of the menace to the interests and safety of the whole British Commonwealth» (2010: 123). Es así que, tanto el crecimiento económico de Estados Unidos como la amenaza nuclear de la Unión Soviética causaron una competencia entre países por la información sobre lo que el enemigo hacía. Como explica Deighton más adelante, la adquisición de información secreta era 
esencial para determinar cuáles serían los movimientos del exterior y cómo estos últimos afectarían la posición de Gran Bretaña en la Guerra Fría. Uno de los grandes problemas durante la obtención de información era que esta corría el riesgo de estar equivocada o incluso de no ser real (2010: 130). El servicio secreto británico fue una de las organizaciones principales durante la lucha por el conocimiento sobre los otros bandos. La inteligencia británica compitió para saber cuáles serían los movimientos a futuro de los demás grupos, por lo que la carrera armamentista fue el foco de atención de los estados socialista y capitalista.

Esta carrera surgió desde la Segunda Guerra Mundial con la detonación de la bomba atómica en Hiroshima. Para los años cincuenta, la bomba de hidrógeno representó una forma de comunicación silenciosa; Estados Unidos, la Unión Soviética e Inglaterra comenzaron a desarrollar pruebas termonucleares en las que demostraron su poder militar y político a las demás potencias. Gran Bretaña, por su parte, entró a la carrera armamentista para demostrar su supremacía ante Estados Unidos y para mantenerse alerta sobre cualquier ataque sorpresa de los soviéticos. David Holloway comenta sobre esta rivalidad: «Each side feared that the other was seeking the capacity to launch a surprise attack and each stressed the importance of preempting such an attack if it appeared to be imminent. Nuclear threats were both a product of the Cold War and a factor contributing to the great tension of those years» (2010: 396). A partir de la década de los cincuenta, las amenazas de una nueva guerra generaron tensión social y paranoia debido a la presión constante de las potencias enemigas por tener supremacía sobre las demás. Año tras año, la innovación militar y la preparación ante cualquier ataque causaron que la lucha por la información sobre los bandos enemigos fuera de vital importancia. Las redes de inteligencia y espionaje fueron figuras clave para informar acerca de cualquier amenaza nuclear de países enemigos.

\section{LA TRAMA DE ESPÍAS: LA SÁTIRA, LA VEROSIMILITUD Y LA CRÍTICA POLÍTICA}

Durante la Guerra Fría, las tramas de espías representaron una respuesta a los problemas internacionales y la decadencia del sistema gubernamental. David Seed (2003: 115) escribe sobre estas historias en «Spy fiction»:

The action is self-evidently political since it involves national rivalries and constantly veers towards a paranoid vision of 'violation by outside agencies' and 'violation of individual autonomy by 
internal agencies'... Espionage fiction became popular in two periods - the turn of the century and the 1960s-when popular anxieties were growing over the credibility of government processes.

Es decir, la trama de espías centraba su atención en los conflictos políticos entre naciones, ya que funcionaba como un escape de la ansiedad y paranoia. El espía es un personaje con agencia que cumple los deseos insatisfechos de la sociedad. La trama constituye un escape de la realidad en la que los lectores tienen acceso a historias semejantes a las que el gobierno intentaba esconder. Al mismo tiempo de que estas historias satisfacían los deseos del público, los autores, como Greene, también decidían insertar una postura política que visibilizara lo que ocurría en su tiempo.

La sátira es el hilo conductor que une las tres obras de Our Man in Havana. Northrop Frye (1990: 224) en Anatomy of Criticism plantea:

Satire demands at least a token fantasy, a content which the reader recognizes as grotesque, and at least an implicit moral standard, the latter being essential in a militant attitude to experience. [...] The satirist has to select his absurdities, and the act of selection is a moral act.

Es justamente el toque grotesco de la sátira lo que promueve un comentario moral por medio de peculiaridades e, incluso, hechos absurdos en la obra. De esta forma, es necesario que haya un objeto que recibirá una crítica por parte de los personajes o del mismo protagonista, quien será el móvil para hacer una denuncia de tipo social o política. El toque grotesco del que habla Frye está presente en esta obra debido a que el autor utiliza la invención como un arma capaz de poner una nación en caos.

La trama de Our Man in Havana trabaja por medio de la verosimilitud del conocimiento. El diccionario de la Real Academia Española define el concepto de verosímil como aquello «que tiene apariencia de ser verdadero» y «que es creíble por no ofrecer carácter alguno de falsedad» ('verosímil'). El papel de Wormold como espía le confiere autoridad sobre la información que dirigirá a sus receptores. Así, el mensaje a transmitir residirá en un umbral de verosimilitud que permitirá a los lectores creerlo o no. En el caso de Our Man in Havana, la autoridad del espía causará una difuminación de los rastros de falsedad o ambigüedad en los reportes de Wormold. El carácter frágil de la información obligará a los receptores a aceptar de forma incuestionable el mensaje proveniente de La Habana. Lindsay Thompson comenta: 
Wormold's institutional affiliation guarantees his veracity: they cannot conceive of somebody fabricating reports for the government. [...] After all, if people accepted as a matter of course that spies routinely lied to their superiors, then readers would have had a difficult time appreciating the novel's humor (2009: 142).

Cuando los agentes en Londres reciben nuevos reportes de Wormold, el contenido de estos pasa por un proceso de adaptación en el que los agentes adhieren su propia realidad al carácter ficticio de la información.

Hacia el final de la obra, Beatrice, la secretaria e interés amoroso de Wormold, explica a este cómo los dibujos de los misiles habían sido aceptados por Londres en un principio:

Then they said he [Hawthorne] ought to have recognized the parts of a vacuum cleaner when he saw [the drawings]. So he said he had, but there was no reason why the principle of a vacuum cleaner might not be applied to a weapon (Greene, 2007: 216).

Es este sentido, al ser el eje central de la obra, Wormold representa la primera fuente de saber que transfiere información a la oficina del servicio secreto en Londres. La autoridad del protagonista como fuente de los hechos obliga a su audiencia a firmar un pacto de verosimilitud en el que aceptarán la «credibilidad» de los hechos recibidos.

Jim Wormold es el sujeto encargado de transferir la información a los altos mandos de la inteligencia británica. Sin embargo, su papel como espía demuestra, finalmente, que la manipulación de la información pone al sujeto en una posición privilegiada. Al inventar reportes, el personaje principal crea verdades que, según William $M$. Chace, convierten al espía en una especie de dios, cuya autoridad obliga a sus lectores a caer en las redes de una atracción en la que el espía tiene el poder de decidir lo que es verdad y lo que es mentira (1990: 160). El acto de espiar se convierte en una forma de dominación sobre los demás personajes que gravitan la trama. Obligado a ser espía, Jim Wormold decide crear su propia información al verse incapaz de conseguirla realmente. Gracias a esto, este personaje toma un papel activo en el que no solo cambia las vidas de quienes lo rodean, sino que también dirige su propia trama como si él mismo fuera el autor del relato. Hacia el final de la novela, las muertes de personas inocentes hacen ver a Wormold las consecuencias de la autoridad y las implicaciones políticas de manipular la realidad ya establecida. 
En cuanto a la producción del texto base -la novela-, como ya se dijo antes, Greene intentaba hacer un comentario satírico meramente de la inteligencia británica. Como tal, la noción del espía y el entrenamiento del protagonista va en aumento conforme transcurre la novela y culmina con el asesinato de Carter, el espía enemigo infiltrado en Cuba y quien tiene la misión de asesinar a Wormold. Al principio de la novela, Hasselbacher y el protagonista hablan sobre el trabajo como vendedor de aspiradoras y la difícil situación económica debido a los caprichos de Milly. El primer diálogo entre los dos hombres construye al protagonista como un hombre ansioso, atareado, planificador de su tiempo y dinero de forma excesiva. El semblante de Wormold es descrito como: «anxious, criss-crossed and fortysh: much younger than Dr Hasselbacher's - the shadow was there already, the anxieties which are beyond the reach of a tranquilizer» (2007: 7-8). Wormold (2007: 9) hace énfasis en la nueva aspiradora «Atomic Pile Cleaner» y habla sobre el miedo de la gente hacia la bomba atómica y la percepción de la gente sobre el tema:

They don't realize that sort of name may go down in the States, but not here, where the clergy are preaching all the time against the misuse of science. Milly and I went to the Cathedral last Sunday -you know how she is about Mass, thinks she'll convert me, I wouldn't wonder. Well, Father Mendez spent half an hour describing the effect of a hydrogen bomb. Those who believe in heaven on earth, he said, are creating a hell -he made it sound that way too-it was very lucid.

Esta primera presentación de Wormold genera un ambiente de mofa. El protagonista muestra más preocupación por su hija y su estabilidad económica que por la tensión y paranoia en la que vivía la sociedad. Así, tanto el contexto histórico como los problemas personales del protagonista se presentan desde las primeras páginas: la evolución de Wormold en un pseudo-espía introducirá el carácter satírico de la obra. Como Frye (1990: 224) explica:

Two things, then, are essential to satire; one is wit or humor founded on fantasy or a sense of the grotesque or absurd, the other is an object of attack. Attack without humor, or pure denunciation, forms one of the boundaries of satire.

Así, la novela cumple la función de hacer un comentario satírico en el que se presentan los dos puntos esenciales. El primero es el sentido grotesco de la mentira como mecanismo en el que la autoridad de Jim Wormold hace que sus lectores sucumban a la 
verosimilitud de sus reportes. El segundo, el objeto de ataque, es el contexto histórico que envuelve la obra, es decir, la Guerra Fría y la amenaza de la bomba como fuente de paranoia que alimenta el carácter iluso de los agentes en Londres y de la sociedad.

\section{Adaptación Cinematográfica: La Habana de Carol ReEd}

Mientras que la novela centra su atención en construir el personaje de Wormold desde la perspectiva del hombre ansioso y preocupado por el bienestar económico de su hija, la película tiene un interés en la construcción del espacio. La secuencia de apertura del filme, por ejemplo, es crucial para apreciar todo lo que sucede en La Habana en la que vive Wormold. Las primeras tomas de la ciudad muestran grandes edificios y la sociedad trabajadora de La Habana. Hombres y mujeres caminan por la calle, piden limosna o coquetean unos con otros. La filmación de la película con formato cinemascope hace que el fondo juegue un papel primordial ya que determina las acciones de los personajes en cuanto a la apreciación de la trama ${ }^{1}$.

La secuencia de apertura de Our Man in Havana muestra una mujer que nada en una piscina; la cámara hace un paneo hacia la izquierda mientras sigue a la mujer. De fondo suena el danzón «La bella cubana», que acentúa el carácter popular de la sociedad retratada en La Habana de los años cincuenta. La iluminación de esta primera secuencia intenta imitar la luz natural. Después, la cámara hace un gran plano general de La Habana (véase fot. 2) y aparece una leyenda en letras blancas que dice: "This film is set in Cuba before the recent revolution». Esta imagen es central para el resto de la película ya que informa al espectador sobre el contexto histórico y político de una manera específica: al igual que en la novela, se deja en claro que todo ocurre en La Habana durante los años cincuenta.

La leyenda desaparece de la pantalla y hay un corte que muestra una calle de La Habana durante el día. Luego, la cámara hace un plano medio de un hombre recostado sobre un costal de comida. Un plano en contrapicada presenta al hombre mientras este se levanta y ve una mujer parada del otro lado de la calle. Después hay un corte y una toma del plano medio en la mujer; esta come una manzana mientras ve al hombre. La posición de la cámara genera un eyeline

\footnotetext{
1 El formato cinemascope, según Stephen Huntley, es una técnica cinematográfica cuya función era la de comprimir la pantalla y mostrar un panorama amplio y horizontal del espacio en el que se encontraban los personajes. La experiencia del espectador se basaba en la visión panorámica de la pantalla, por lo que su atención hacía un énfasis de la relación de los personajes con el espacio en que se encontraban (1993: 298).
} 
match tanto del hombre como de la mujer. De nuevo un corte muestra un plano medio del hombre; a continuación, un travelling sigue al hombre mientras cruza la calle y camina hacia la mujer. Un plano de dos (two shot) exhibe cómo el hombre y la mujer se encuentran. El hombre se acerca más a la mujer; esta le lanza la manzana; él toma la fruta y la huele. Acto seguido, la toma se congela (freeze frame) y comienzan a aparecer los nombres de los actores de la película y, finalmente, el título: Our Man in Havana.

Posteriormente, la cámara se descongela; el hombre y la mujer caminan el uno junto al otro; los dos desaparecen en la calle. En el mismo plano aparece Hawthorne, un tracking shot sigue a este mientras camina por la avenida. La toma se centra en un auto; dentro del auto, un plano medio muestra al capitán Segura, quien sigue a Hawthorne mientras camina por la acera. Hawthorne se dirige a la tienda de Wormold. A esta escena de acecho le sigue un corte y un plano de dos del doctor Hasselbacher y Wormold. Hawthorne ve a los dos hombres y camina hacia ellos. Hawthorne entra a la tienda; Wormold se despide de Hasselbacher y también entra. El protagonista y Hawthorne comienzan a hablar. Wormold pregunta si este último quisiera comprar una aspiradora.

Otro corte presenta un plano medio de un policía. Detrás de él está el auto del capitán Segura. El policía llama a Hasselbacher, quien camina por la calle después de despedirse de Wormold. El policía pregunta al hombre por sus papeles; este, desconfiado, se rehúsa y se dirige al auto de Segura, quien sigue dentro del auto. La cámara hace una toma por encima del hombro de Hasselbacher inclinado hacia la ventanilla del auto; Segura pide los papeles del doctor y este se los da. Segura le pregunta si conoce al hombre inglés que caminaba por la calle hace unos momentos (Hawthorne). Un primer plano muestra el semblante desconfiado de Hasselbacher, quien argumenta no conocer al hombre. Acto seguido, la cámara hace un primer plano de Segura, quien, insatisfecho, le devuelve los papeles al médico y se va.

Enseguida hay un corte y la cámara abre de nuevo en la tienda de aspiradoras. Un plano de dos exhibe a Hawthorne y Wormold mientras hablan. A su vez, Hawthorne, en su papel de espía encubierto, indaga sobre los asuntos personales de Wormold mientras este se mueve por la tienda, presenta la aspiradora a Hawthorne y responde sus preguntas de forma vaga. Luego la cámara hace una toma por encima del hombro de Wormold, quien mira a Hawthorne. Este se despide después de hacer su cuestionario y dice: «l'll be seeing you again. Here or there». Después, Hawthorne desaparece de la escena. 
Como se describió antes, la primera escena de la película muestra un plano general de La Habana y también el año en el que esta se filmó, por lo que la atención del espectador cae sobre el espacio y el contexto sociopolítico de la película. La cámara hace un uso constante de los paneos y del tracking shot de los personajes; así pues, en el fondo siempre están a la vista las calles de La Habana y la gente que la habita. Hay una descripción visual de los vendedores, músicos, bares y la pobreza de la gente (véase fot. 3). Así, las primeras escenas de la película establecen el aspecto histórico de la película por encima de construir el personaje de Wormold, quien no aparece en cámara sino hacia el final de la primera escena.

Las escenas de La Habana exponen el panorama que se vivía en los años cincuenta con el régimen de Fulgencio Batista y los abusos de la policía. Este aspecto es un punto importante dentro de la novela, pero la diferencia recae en que el narrador hace énfasis en los personajes más que en lo que los rodea. La trama de la novela pone en primer lugar la construcción de Wormold como un hombre común que se convierte en espía para el servicio británico y cuyas mentiras desatan un caos para Inglaterra durante la Guerra Fría.

La primera secuencia incluye la relación de la policía con la gente de Cuba. La aparición del capitán Segura en el auto demuestra el temor de la gente hacia la imagen imponente de Segura. Durante la persecución de Hawthorne, la música incidental se torna de misterio e incertidumbre mientras un hombre se aproxima al auto del capitán y grita en español: «Canalla, miserable, asesino. Ya verás, deja que esto se caiga, la vas a pagar» (véase fot. 4). Este primer comentario denota una posición política contra el gobierno de Fulgencio Batista. A manera de contraste, la banda sonora de la película, también en español, ayuda a construir el contexto sociopolítico del filme. Mientras que la música representa la Cuba que ven los turistas (seducción, ritmo, constante movimiento y alegría), los diálogos que ocurren de fondo por parte de la gente cubana tienen la función de mostrar las preocupaciones de esa sociedad durante los años cincuenta. El hombre que decide lanzarse hacia el auto de Segura es la representación del pueblo. Esta escena se convierte en una sinécdoque: la acción de confrontar a la policía indicará el deseo de revolución y futura rebelión de los cubanos hacia el régimen de Batista $^{2}$. En otro momento de la película, Wormold camina por la

\footnotetext{
${ }^{2}$ Según el diccionario de la Real Academia Española, «sinécdoque» es una figura retórica que indica el intercambio de una cosa con el nombre de otra. Una de sus características es que una parte tomará la posición del todo o viceversa.
} 
calle y una voz de fondo dice la siguiente línea: «La revolución es lo único que puede vencer esto. Mira, Batista todos los días está matando gente en la calle. Los periódicos censurados. ¿Tú qué te crees?». Así, la voz del pueblo vuelve a escucharse con un mensaje indirecto, que, al estar en español, difícilmente será entendido por los turistas, pero que indica la represión y miedo de la gente a las políticas del gobierno cubano de mediados del siglo XX.

Asimismo, la gente en las calles se aproxima a Hawthorne para ofrecerle música, recorridos por la ciudad, e incluso mujeres. La música violenta que caracterizaba a Segura ahora cambia a la canción «¿Dónde vas, Domitila?», lo que, de nuevo, introduce el ambiente popular en el filme. Esta canción vuelve a oírse durante el resto de la película y se transformará en un motivo recurrente que suena mientras la cámara hace paneos de la ciudad o, incluso, de los burdeles de La Habana.

Es así que el contexto político de Cuba se convierte en una constante durante la película. Los diálogos y la presentación del fondo en el que se desarrollan los personajes funcionan como un móvil en el que, además de presentar una trama de espías, también se espera que haya un comentario sutil sobre el aspecto de la Cuba prerevolucionaria.

\section{Adaptación teatral: La Habana de Clive Francis}

El tercer medio a analizar de Our Man in Havana es una obra de teatro con el mismo nombre y adaptada por Clive Francis en dos actos. Esta obra se caracteriza por recrear la historia de sucesiones rápidas cuyo único fin es crear una trama de enredos centrada en los personajes. Las acciones de Wormold como espía tienen como fin la resolución de los problemas generados por el encadenamiento de la serie de reportes ficticios. Es decir, el contexto histórico y los comentarios hacia la Guerra Fría quedan minimizados por las acciones de los personajes. La guía de estudio de la compañía de teatro Vertigo explica sobre la adaptación: «In Clive Francis' adaptation, Graham Greene's classic satirical novel becomes a wonderfully funny and fastmoving romp. Our Man in Havana is a play with dozens of characters played by only four actors» (2016: 7). Así, las veintisiete escenas del acto uno y las dieciocho escenas del acto dos ocurren de una forma vertiginosa: la sucesión de hechos en la trama y la creación de momentos cómicos se hallan en una posición privilegiada. Los cuatro actores tienen la misión de cambiar de vestuario y posiciones en cada 
una de las escenas, por lo que es indispensable mantener un diálogo corto, rápido y constante para que el espectador sea capaz de seguir la trama.

La primera escena de la obra menciona la situación sociopolítica de Cuba y el año en el que está insertada la trama, por medio de narradores extradiegéticos que mencionan los aspectos importantes de la Cuba de Batista durante los años cincuenta:

3. NARRATOR: Havana was always a city to visit, never a city to live in.

4. NARRATOR: Where every vice was permissible and every trade was possible.

2. NARRATOR: This was the Cuba Wormold first came to. Where hope seemed as hopeless as trying to sell vacuum cleaners...

3. NARRATOR: And where the impossible was possible, if you knew the right person at the appropriate price (Francis, 2015: 1.1).

Aunque haya una presentación del contexto histórico de la trama desde el acto uno, la obra de teatro parece prescindir de una presentación detallada acerca de la presencia de Wormold en La Habana. El rápido diálogo de los personajes no permite una apreciación del entorno ni del contexto en el que se encuentran los personajes. Más adelante, la escena dos del primer acto introduce a Wormold y a su ayudante, López, en la tienda. El primer diálogo de estos personajes gira en torno a la extraña presencia de un hombre inglés en la tienda (Hawthorne). Seguidamente, los dos ingleses proceden a hablar del negocio y a responder preguntas personales acerca del vendedor. De esta forma, las primeras dos escenas introducen la trama de la historia de una forma rápida: Hawthorne interroga a Wormold; los dos hombres se despiden y el protagonista queda en un estado de confusión. Más adelante, en la escena seis del acto uno, Hawthorne obliga al personaje principal a aceptar el trabajo, lo cual lo deja perplejo e incapaz de negarse:

HAWTHORNE: Don't be difficult. I'm having traces of him sent out from London. A good tip -if you run out of ink use bird shit, am I going too fast?

WORMOLD: I haven't even said I was willing.

HAWTHORNE: London agrees to pay $\$ 150$ a month, with another hundred and fifty as expenses - you'll have to justify those of course (Francis, 2015: 1.6).

La trama no se detiene y las acciones principales de la obra tienen un toque cómico que inclinan a la obra al género de la farsa y la alejan 
de la sátira. Chris Bladick en el Concise Oxford Dictionary of Literary Terms define la farsa como «a kind of comedy that inspires hilarity mixed with panic and cruelty in its audience through an increasingly rapid and improbable series of ludicrous confusions, physical disasters, and sexual innuendos among its stock characters» ('Farce'). La adaptación teatral de Our Man in Havana, en efecto, exhibe una trama de acción más que ser una que induzca a una moraleja. El veloz intercambio de líneas tiene como motivo el hacer comentarios forzados en los que la sucesión de eventos absurdos acompaña una trama de confusiones donde el contexto histórico queda relegado o incluso olvidado. El uso de acontecimientos absurdos es un elemento sustancial en la farsa ya que exalta el cómo los personajes intentan resolver un predicamento que da inicio a la trama. Sin embargo, la búsqueda de esta resolución inducirá a la creación de un encadenamiento de acciones fuera de lo común, que culminará de una forma improbable y exagerada. Este último aspecto difiere del elemento grotesco del que habla Frye porque la sátira tiene el motivo primigenio de hacer un comentario moral como razón de la creación de la trama. La farsa, por otro lado, mantiene la exageración y el absurdo al nivel de los personajes y el seguimiento de acciones en el argumento.

Un evento que desemboca en lo absurdo se presenta en las escenas finales de la obra. Hawthorne cita a Wormold en su oficina para informarle que Londres ha descubierto su red de mentiras. Para evitar la ridiculización del servicio secreto al exponer al público los hechos inexistentes que casi ocasionan una nueva guerra mundial, los altos mandos deciden ocultar los verdaderos motivos de Wormold. El servicio secreto lo contrata como conferencista y lo premian como oficial de la Orden del Imperio Británico. Aunque estos hechos también ocurren en la obra original, la obra teatral decide llevar este último acto cómico al extremo y hace que la misma reina interrumpa la escena y condecore a Wormold:

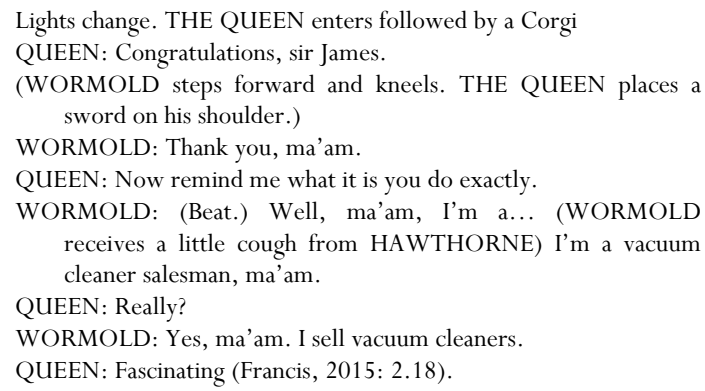


Como ya mencionado, según Frye, hay dos instancias para hacer sátira: humor basado en lo grotesco o absurdo de algún evento específico y un objeto de ataque que llevará la trama hacia un comentario moral. Sin embargo, la adaptación de Francis retoma la trama absurda de la obra original y relega el objeto de ataque a un segundo plano. Hay una mención del carácter histórico de la obra en la primera escena, pero su función es, simplemente, la de contextualizar una comedia en la que hay una presentación de humor grotesco y eventos absurdos, cuyo único fin es el cómico.

\section{CONCLUSIONES}

En conclusión, con el estudio de una obra en dos medios diferentes queda demostrado cómo la adaptación es un proceso de re-interpretación en el que el adaptador se ocupa de los aspectos que más le interesen de una obra con el motivo de reescribir una nueva lectura de la postura original. El autor que decide adaptar un texto toma los aspectos relevantes de la obra original, pero cambia la perspectiva primera con la que se concebía la trama. Las tres obras estudiadas utilizan el modelo de la trama de espías como base para hacer tres tipos de comentarios distintos. En primer lugar, la novela sigue las convenciones de la sátira, ya que establece una dicotomía entre el personaje principal y la trama en la que se circunscribe. El acto grotesco de la trama muestra cómo Jim Wormold se convierte en un pseudo-espía que utiliza la invención para tomar agencia en la trama y dirigirla hacia sus fines personales. Así, los conceptos de verdad y ficción quedan diluidos debido, principalmente, a la credulidad de los agentes ingleses que reciben sus reportes. Como resultado, el comentario moral de la trama indica las fallas en el servicio secreto británico en cuanto al manejo de información durante la Guerra Fría.

En segundo lugar, la película de Carol Reed, a diferencia de la novela, aunque también guarda relación con el comentario satírico del manejo de información, se vale del modelo del pseudo-espía para presentar el trasfondo político de Cuba por medio de los espacios. La situación de pobreza y corrupción vivida en Cuba durante el gobierno de Fulgencio Batista reencarnará en el personaje del Capitán Segura, quien aparece desde el inicio de la película y dirige la trama hacia una presentación de las injusticias de la policía cubana. Así, el ambiente de tensión que se vivía en Cuba a finales de la década de los cincuenta opacará el comentario satírico acerca de la inteligencia británica. Finalmente, la obra de Clive Francis sale del género satírico para entrar en la farsa. La adaptación no cumple con la dicotomía de la 
sátira debido a que se toma la trama cómica de la obra original para convertirla en una sucesión de eventos absurdos en los que el contexto histórico queda relegado a un segundo plano.

Las dos adaptaciones carecen del balance satírico de la novela. La obra base trabaja con una dicotomía en la que el personaje principal funciona para conducir al lector a un comentario moral. Las dos adaptaciones, no obstante, rompen con esa dicotomía y optan por trabajar con sus elementos por separado. En el caso de Carol Reed, el contexto histórico llega a tener más peso que el del crecimiento de Wormold como creador de sus propias verdades. Clive Francis decide, por otra parte, diluir el contexto histórico para darle más peso a la relación de los personajes con los eventos absurdos de la trama, por lo que cae en el género de farsa. Así se demuestra cómo la literatura es un género maleable que permite la constante transformación de tramas y personajes a gusto del adaptador. La relación entre obra base y secundaria indica cómo la adaptación exige la reinterpretación y la segunda lectura con el propósito de satisfacer a distintos y nuevos tipos de audiencia de acuerdo con su contexto histórico.

\section{IMÁGENES}

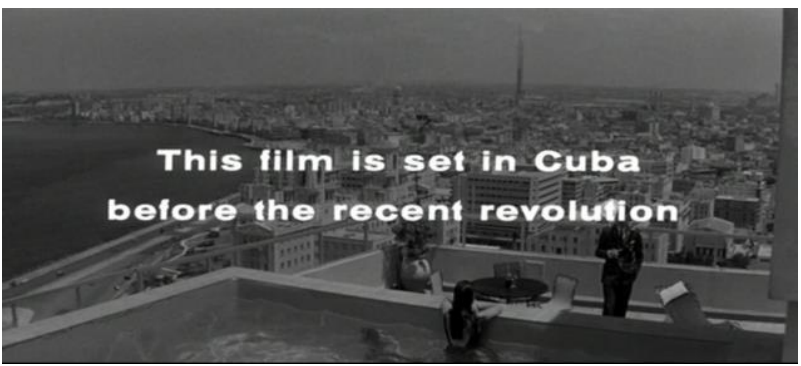

Fot. 1. Escena de apertura de Our Man in Havana (1959). 
Blanca Herrmann Estudillo

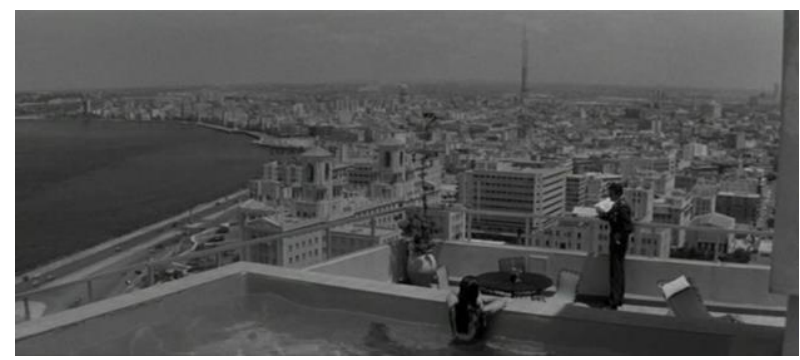

Fot 2. Uso de la técnica Cinemascope para dar privilegio a los fondos y espacios de la trama.

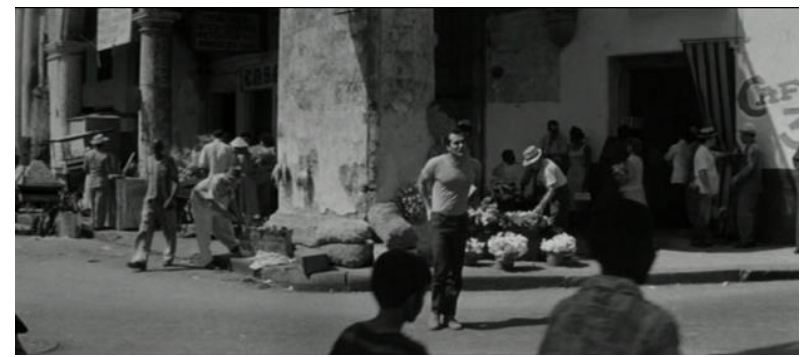

Fot. 3. La vida de la gente trabajadora en La Habana.

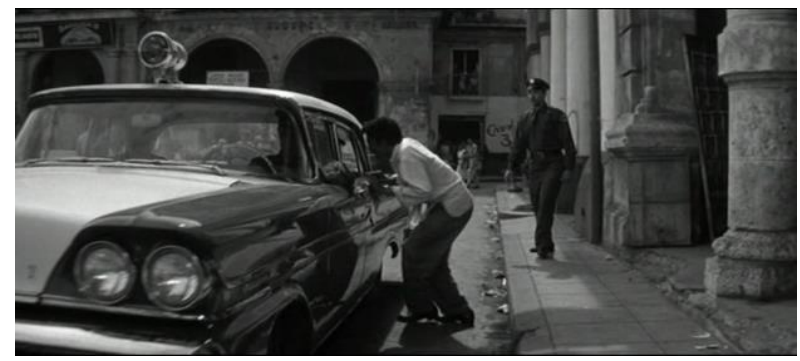

Fot. 4. Un hombre confronta al capitán Segura. Al fondo, un policía arrastrará al hombre y lo golpeará. 


\section{BIBLIOGRAFÍA CITADA}

BENZ, Stephen (1988), «Graham Greene and Cuba: A Commitment», Confluencia, 4/1, págs. 109-117 [En línea www.jstor.org/stable/27921813. Fecha de consulta: 4/05/2021]. Brennan, G. Michael (2010), «Chapter 6. The Writer in Search of New Directions: 1955-1965», Graham Greene: Fictions, Faith and Authorship, Londres, Continuum Books, págs. 103-119.

Carroll, Jacob (2009), Authors of Truth Writers, Liars, and Spies in Our Man in Havana, Haverford College [Tesis Doctoral].

Chace, M. William (1990), «Spies and God's Spies: Greenes's Espionage Fiction», en Jeffrey Meyers (ed.), Graham Greene: A Revaluation, Nueva York, Palgrave Macmillan, págs. 156-181.

CONCISE OXFORD DiCTIONARY OF LITERARY TERMS, THE (2015), Nueva York, Oxford University Press.

Deighton, Anne (2010), «Britain and the Cold War, 1945-1955», en Melvyn P.Leffler y Odd Arne Westad [eds.], The Cambridge History of the Cold War: Volume 1: Origins, Cambridge, Cambridge University Press, págs. 112-132.

Francis, Clive (2015), Our Man in Havana, Londres, Oberon Books.

FRYE, Northrop y Robert D. DENHAM (1990), «THIRD ESSAY. Archetypal Criticism: Theory of Myths: The Mythos of Winter: Irony and Satire», Anatomy of Criticism: Four Essays, Oxfordshire, Princeton University Press, págs. 223-43.

Greene, Graham (2007), Our Man in Havana, Nueva York, Penguin Books.

Hulme, Peter (2008), «Graham Greene and Cuba: Our Man In Havana?», NWIG: New West Indian Guide / Nieuwe West-Indische Gids, 82/3/4, págs. 185-209 [En línea: www.jstor.org/stable/43390733. Fecha de consulta: 4/05/2021]. Holloway, David (2010), «Nuclear weapons and the escalation of the Cold War, 1945-1962», en Melvyn P. Leffler y Odd Arne Westad (eds.), The Cambridge History of the Cold War: Volume 1: Origins, Cambridge, Cambridge University Press, págs. 376-397.

HunTley, Stephen (1993), «Sponable's CinemaScope: An Intimate Chronology of the Invention of the CinemaScope Optical System», Film History, 5/3, págs. 298-320 [En línea: www.jstor.org/stable/3815144. Fecha de consulta: 4/05/2021].

HutcheON, Linda (2013), «Beginning to Theorize Adaptation: What? Who? Why? How? Where? When?», A Theory of Adaptation, Nueva York y Londres, Routledge, págs.1-32.

Diccionario DE LA LENGUA ESPAÑOLA (2021) [En línea: https://dle.rae.es/] 
Blanca Herrmann Estudillo

SeED, David (2003), «Spy Fiction», en Martin Priestman (ed.), The Cambridge Companion to Crime Fiction, Nueva York, Cambridge University Press, págs. 115-135.

Study Guide: Graham Greene's Our Man in Havana (2016), Vertigo Theatre, Alberta, Pembina.

THOMPSON, Brian Lindsay (2009), «Our Man in Havana and Auteurism», Graham Greene and the Politics of Popular Fiction and Film, Hampshire, Palgrave Macmillan, págs. 135-155.

Fecha de recepción: 14/02/21.

Fecha de aceptación: 19/04/21. 